Ann. Biol. anim. Bioch. Biophys., I963, 3 (3), 299-305

\title{
EFFETS DE LA PYRIDOXINE SUR LE NIVEAU PORTAL DES ACIDES AMINÉS APRÈS UN REPAS PROTÉIQUE
}

\author{
S. SZMELCMAN et K. GUGGENHEIM \\ Laboratoire de Nutrition, Département de Biochimie, \\ École de Médecine de l'Université Hébraïque et d'Hadassah, Jérusalem (Israël)
}

SOMMAIRE,

L'action de la déoxypyridoxine sur le niveau portal des acides aminés - lysine, méthionine et tryptophane - a été analysée chez des rats normaux après un repas de lactalbumine ou après l'ingestion d'acides aminés isolés. Il a été constaté que cette action est surtout sensible sur les niveaux du tryptophane et de la lysine, la teneur de la méthionine ne paraissant pas modifiée.

L'apyridoxinose provoque une baisse de la teneur en lysine et en tryptophane seulement, tandis que l'administration de la pyridoxine n'annule pas l'action de l'avitaminose sur le tryptophane.

L'amidon, à certaines concentrations, trouble l'effet de la déoxypyridoxine.

\section{INTRODUCTION}

Le problème de l'absorption intestinale en général, et des acides aminés en particulier, bien que souvent abordé, reste néanmoins à élucider. De nombreuses recherches ont permis cependant d'entrevoir les prémices du mécanisme régissant l'absorption.

Il est actuellement acquis que de nombreux acides aminés, surtout les formes L, sont absorbés d'une façon active (AGAR, HIRD et SidHU, I953). En partant de cette constatation et en situant le mécanisme intime de l'absorption au niveau cellulaire, il est possible d'apprécier les résultats de CHRISTENSEN et al. (I954) et de Fridhandler et QUASTEL, (I955) comme étant une contribution logique à la solution du problème. Il apparaît ainsi que la pyridoxine joue un rôle actif dans l'absorption des acides aminés, et que les antimétabolites, déoxypyridoxine et 
DNP (1), possèdent un pouvoir inhibiteur (JACOBS et LUPER, I957; JACOBS et al., I960).

Ia plupart des recherches réalisées soit in vitro (AkEDo et al., I960), soit in situ (JACOBS, I962) avec des acides aminés isolés et non avec des protéines naturelles, présentent l'inconvénient de s'écarter de l'état physiologique, réduisant ainsi la portée des résultats obtenus.

Au cours du présent travail, nous étudierons l'effet de la pyridoxine sur le niveau portal des acides aminés, effet qui est probablement en rapport avec le mécanisme de l'absorption intestinale. La technique de ce travail, déjà utilisée antérieurement (GUGGENHEIM et al., I960; Gol,DBERG et GUGGENHEIM, I962), répond judicieusement, pensons-nous, au besoin ressenti de serrer de plus près les conditions naturelles et physiologiques.

\section{MATÉRIEL E'T MÉTHODES}

Nos expériences sont effectuées sur des rats "albinos " de sexe mâle dérivant d'une souche

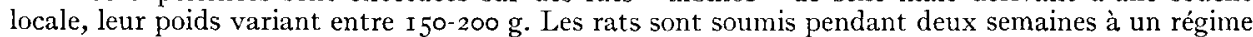
de base dont la composition est décrite dans un mémoire antérieur (Cruggenherm et al., 1960). A la fin de cette période, les rats sont laissés à jeun pendant 48 heures. Ce long jeûne est jugé indispensable pour garantir l'évacuation totale des résidus alimentaires de la lumière intestinale.

Un repas protéique est alors administré aux rats par sonde stomacale ; ce repas, constitué de $5 \mathrm{p}$. 100 de lactalbumine et de $5 \mathrm{p}$. 100 d'amidon en solution aqueuse, est fourni aux rats sous forme de ration de $5 \mathrm{ml}$ pour $100 \mathrm{~g}$ de poids corporel. Un groupe de contrôle reçoit uniquement une solution de 5 p. Ioo d'amidon. On donne à une série de rats des acides aminés purs à la place de la protéine native. Les quantités de lysine, méthionine et tryptophane fournis aux rats correspondent aux concentrations de ces mêmes acides aminés dans $5 \mathrm{~g}$ de lactalbumine. La déficience chez les rats est obtenue grâce au régime de base (GUGGENHEIM et al., I960) dépourvu de pyridoxine et, pour accélérer l'avitaminose, on ajoute $20 \mathrm{mg}$ de déoxypyridoxine par $\mathrm{kg}$ d'aliment.

La détermination quantitative des acides aminés du sang portal est réalisée suivant une méthode microbiologique décrite en détail par BARTON-WRIGHT (BARTON-WRIGHT, I952). Le sang est prélevé 30-60 minutes après le repas de lactalbumine et ro minutes après l'administration des acides aminés isolés, ces intervalles de temps correspondant aux concentrations maximales des acides aminés dans le plasma portal. Les rats reçoivent simultanément avec le repas protéique une dose de $5 \mathrm{mg}$ de déoxypyridoxine par roo $\mathrm{g}$ de poids corporel.

\section{RÉSULTATS}

I. La première étape de nos expériences est résumée dans le tableau I. L'interprétation des résultats réunis dans ce tableau nous permet d'aboutir aux conclusions suivantes :

a) L'action de l'antimétabolite déoxypyridoxine sur les rats normaux est à peine sensible sur le niveau portal de la lysine; elle n'est pas du tout ressentie sur le niveau de la méthionine. Par contre, l'influence de la déoxypyridoxine est très nette sur la concentration plasmatique du tryptophane.

(1) 2,4-Dinitrophénol. 
b) La déficience en pyridoxine provoque une baisse de concentration de la lysine; elle paraît sans effet sur le nivea1 de la méthionine; elle est très sensible sur le tryptophane.

\section{TABLEAU I}

Teneur en lysine, méthionine et tryptophane du plasma portal chez des rats normaux et déficients en pyridoxine, après un repas de lactalbumine ou d'amidon

\begin{tabular}{|c|c|c|c|c|c|c|c|c|}
\hline \multirow{4}{*}{$\begin{array}{l}\text { Repas } \\
\text { fourni } \\
\text { aux rats }\end{array}$} & \multicolumn{4}{|c|}{ Rats normaux } & \multicolumn{4}{|c|}{ Rats déficients } \\
\hline & \multicolumn{2}{|c|}{ Contrôle } & \multicolumn{2}{|c|}{$\begin{array}{c}\text { Traités à la } \\
\text { déoxypyridoxine }\end{array}$} & \multicolumn{2}{|c|}{ Contrôle } & \multicolumn{2}{|c|}{$\begin{array}{l}\text { Traités à la } \\
\text { pyridoxine }\end{array}$} \\
\hline & \multicolumn{4}{|c|}{ Temps après ingestion (en minutes) } & \multicolumn{4}{|c|}{ Temps après ingestion (en minutes) } \\
\hline & 30 & 60 & 30 & 60 & 30 & 60 & 30 & 60 \\
\hline
\end{tabular}

L-lysine

\begin{tabular}{|c|c|c|c|c|c|c|c|c|}
\hline $\begin{array}{l}\text { Lact- } \\
\text { albumine }\end{array}$ & $\frac{122+4,67}{(10)}$ & $\begin{array}{c}128 \pm 4,41 \\
(10)\end{array}$ & $110 \underset{(9)}{ \pm} 5,52$ & $116 \underset{(9)}{ \pm} 5,25$ & $100 \underset{(7)}{ \pm} 6,91$ & $\begin{array}{c}97 \pm 4,25 \\
(7)\end{array}$ & $110 \frac{1}{(7)}^{4}, 25$ & $120 \pm \frac{1}{(7)} 5$ \\
\hline Amidon & $60 \underset{(7)}{ \pm} 3,09$ & $71 \underset{(7)}{ \pm} 3,40$ & $70 \underset{(7)}{ \pm} 4,89$ & $70 \underset{(7)}{ \pm}, 4,36$ & $72 \underset{(7)}{ \pm} 3,1$ & $60 \underset{(7)}{ \pm} 3$ & $68 \underset{(7)}{ \pm} 2,7$ & $70 \underset{(7)}{ \pm} 2,5$ \\
\hline
\end{tabular}

L-méthionine

\begin{tabular}{|c|c|c|c|c|c|c|c|c|}
\hline $\begin{array}{l}\text { Lact- } \\
\text { albumine }\end{array}$ & $13 \underset{(8)}{ \pm} 0,75$ & $14 \underset{(9)}{ \pm} 0,57$ & $12 \underset{(7)}{ \pm} 1,07$ & $14 \frac{ \pm}{(9)} 0,95$ & $15 \underset{(6)}{ \pm} 1,10$ & $15 \underset{(6)}{ \pm} 1,25$ & $15 \underset{(6)}{ \pm} 0,75$ & $14 \underset{(6)}{ \pm} 1$ \\
\hline Amidon & $11 \underset{(7)}{ \pm} 0,37$ & $10 \underset{(7)}{ \pm} 0,34$ & $10 \underset{(7)}{ \pm} 0,59$ & $10 \underset{(7)}{ \pm} 0,51$ & $12 \underset{(7)}{ \pm} 0,37$ & $10 \underset{(6)}{ \pm} 0,34$ & $11 \underset{(6)}{ \pm} 0,40$ & $11 \underset{(6)}{ \pm} 0,36$ \\
\hline
\end{tabular}

L-tryptophane

\begin{tabular}{|c|c|c|c|c|c|c|c|c|}
\hline $\begin{array}{l}\text { Lact- } \\
\text { albumine }\end{array}$ & $40 \frac{ \pm}{(9)} 2,85$ & $47 \pm 2,35$ & $32 \frac{t}{(10)} 1,67$ & $36 \underset{(10)}{ \pm} 2,32$ & $31 \underset{(6)}{ \pm} 1,35$ & $35 \pm \frac{ \pm}{(6)} 1$ & $3^{\prime} \underset{(6)}{ \pm} 2$ & $32 \underset{(6)}{ \pm} 1,1$ \\
\hline Amidon & $31 \pm 0,73$ & $31 \underset{(7)}{ \pm} 0,73$ & $26 \underset{(7)}{ \pm} 0,75$ & $30 \underset{(7)}{ \pm} 0,43$ & $30 \underset{(6)}{ \pm} 0,80$ & $31 \underset{(6)}{ \pm} 0,70$ & $30 \underset{(6)}{ \pm} 0,5$ & $28 \underset{(6)}{ \pm} 0,7$ \\
\hline
\end{tabular}

Remarques : Les résultats sont exprimés en $\mu \mathrm{g}$ par $\mathrm{ml}$ de plasma.

Les valeurs indiquées sont les valeurs moyennes et les erreurs standard sur la moyenne.

Les nombres entre parenthèses expriment le nombre de rats par expérience. 
c) L'addition de la pyridoxine aux rats déficients entraîne une montée du niveau de la lysine; elle reste sans effet sur la méthionine et sur le tryptophane.

II. La deuxième étape de ce travail nous a conduits à vérifier la concentration des trois acides aminés étudiés chez des rats à jeun avant et après addition de la déoxypyridoxine. Les valeurs obtenues sont évidemment des valeurs de contrôle (tableau 2).

TABLEAU 2

Effets de la déoxypyridoxine sur le niveau des acides aminés du plasma portal chez des rats à jeun, avant et $10 \mathrm{mn}$ après l'administration de ces acides aminés

\begin{tabular}{|c|c|c|c|c|c|c|}
\hline \multirow{2}{*}{$\begin{array}{l}\text { Acides aminés } \\
\text { analysés }\end{array}$} & \multicolumn{3}{|c|}{ Avant l'administration } & \multicolumn{3}{|c|}{10 minutes après l'administration } \\
\hline & Lysine & Méthionine & Tryptophane & Lysine & Méthionine & Tryptophane \\
\hline Contrôle & $74 \underset{(5)}{ \pm} 2,45$ & $8,80 \underset{(5)}{\frac{1}{(5)}} 0,19$ & $17 \underset{(5)}{ \pm} 0,80$ & $247 \underset{(9)}{ \pm} 8,66$ & $47 \underset{(8)}{ \pm} 2,42$ & $129 \underset{(8)}{ \pm} 10,2$ \\
\hline $\begin{array}{l}\text { Déoxypyri- } \\
\text { doxine per os }\end{array}$ & $72 \underset{(5)}{ \pm} 3,74$ & $8,2 \underset{(5)}{ \pm} 0,30$ & $19 \underset{(5)}{ \pm} 1$ & $229 \underset{(8)}{ \pm} 13,12$ & $39 \underset{(8)}{ \pm} 2,98$ & $73 \underset{(9)}{ \pm} 3,91$ \\
\hline $\begin{array}{l}\text { Déoxypyri- } \\
\text { doxine } \\
\text { injection intra- } \\
\text { péritonéale }\end{array}$ & - & 一 & 一. & $232 \underset{(6)}{ \pm} 12,48$ & $31 \underset{(6)}{ \pm} 1,95$ & $83 \underset{(7)}{ \pm} 3,91$ \\
\hline
\end{tabular}

Remarques : La concentration des acides aminés fournis aux rats est équivalente à celle se trouvant dans $5 \mathrm{~g}$ de lactalbumine.

Les résultats sont exprimés en $\mu \mathrm{g}$ par ml de plasma.

Les valeurs indiquées sont les valeurs moyennes et les erreurs standard sur la moyenne.

Les nombres entre parenthèses expriment le nombre de rats par expérience.

Nous voyons - comme cela était d'ailleurs prévisible -- que la déoxy pyridoxine reste sans effet sur ces niveaux de base. Nous avons également voulu vérifier l'action de la déoxypyridoxine sur le niveau portal des trois acides aminés étudiés après leur administration perorale à la place de la lactalbumine.

Les résultats du tableau 2 nous montrent que l'effet de la déoxypyridoxine est ressenti sur la méthionine et le tryptophane, mais ne l'est pas sur la lysine. Il faut remarquer que l'antimétabolite injecté par voie intrapéritonéale ou par voie perorale présente une activité similaire, plus accentuée après l'ingestion des acides aminés isolés. De plus, dans ce cas, les valeurs absolues des acides aminés au niveau portal sont plus élevées, bien que les concentrations initiales des acides aminés fournis aux rats soient les mêmes dans le cas du repas de lactalbumine ou de l'administration des acides aminés isolés.

Ces dernières remarques laissent supposer que la présence des substances autres que les acides aminés analysés influe sur le niveau portal de ceux-ci, ainsi que sur l'ampleur de l'activité de la déoxypyridoxine. C'est pourquoi nous avons étudié l'action de l'antimétabolite sur le tryptophane dilué dans des concentrations 
différentes d'amidon. D'après les résultats du tableau 3, nous voyons que l'amidon à des concentrations élevées de $0,50 \mathrm{~g}$ par Ioo $\mathrm{g}$ de rat, tout en abaissant les valeurs absolues du trytpophane, trouble, sinon annule, l'effet de la déoxypyridoxine.

TABIEAU 3

Influence de l'amidon et de la déoxypyridoxine sur le niveau portal du tryptophane après l'administration de l'acide aminé par sonde stomacale (en ug par ml de plasma)

\begin{tabular}{|c|c|c|}
\hline \multirow{2}{*}{$\begin{array}{l}\text { Quantité } \\
\text { d'amidon }\end{array}$} & \multicolumn{2}{|c|}{ Teneur en tryptophane } \\
\hline & Rats témoins & $\begin{array}{l}\text { Rats soumis à la } \\
\text { déoxypyridoxine }\end{array}$ \\
\hline - & $129 \underset{(8)}{ \pm} 10,20$ & $79 \underset{(9)}{ \pm} 3,91$ \\
\hline $\begin{array}{l}0,25 \mathrm{~g} \text { par } 100 \mathrm{~g} \\
\text { de rat }\end{array}$ & $148 \pm 3,3,58$ & $116 \underset{(5)}{ \pm} 9,50$ \\
\hline $\begin{array}{l}0,50 \mathrm{~g} \text { par } 100 \mathrm{~g} \\
\text { de rat }\end{array}$ & $113 \underset{(6)}{ \pm} 4,52$ & $107 \underset{(6)}{ \pm} 6,70$ \\
\hline
\end{tabular}

Remarques : Les valeurs indiquées sont les valeurs moyennes et les erreurs standard sur la moyenne. Les nombres entre parenthèses expriment le nombre de rats par expérience.

\section{DISCUSSION}

Nous nous sommes assigné pour but dans ce travail la recherche des conditions se rapprochant le plus de l'état physiologique, afin que les résultats obtenus aient une valeur maximale. En tenant compte des travaux réalisés dans notre laboratoire et du fait que les acides aminés, après leur absorption intestinale, sont acheminés par la voie portale vers le foie, nous avons utilisé une technique qui semble plus judicieuse que les expériences in vitro ou in situ.

Au cours de nos expériences, nous avons voulu mettre en évidence l'influence de la pyridoxine sur le niveau portal des trois acides aminés : 1ysine, méthionine et tryptophane. D'après les résultats obtenus, nous voyons que l'action antagoniste de la déoxypyridoxine n'est pas également ressentie sur le niveau portal des trois acides aminés. Dans toutes les conditions expérimentales utilisées, seule la teneur portale du tryptophane est sensible de façon nette et répétée à l'action de l'antimétabolite; la concentration de la méthionine paraît par contre la plus stable et la moins influençable. L'avitaminose prolongée des rats agit incontestablement sur le niveau du tryptophane et de la lysine, tandis que l'addition perorale de la pyridoxine n'annule pas l'effet de l'antimétabolite sur la concentration du tryptophane et provoque une légère remontée du niveau dela lysine. Il est probable que le temps d'action laissé à la pyridoxine était insuffisant pour supprimer un état de déficience accentué. 
Cette première partie expérimentale ayant été réalisée avec la lactalbumine comme source d'acides aminés, nous avons voulu administrer aux rats des acides aminés purs et comparer les résultats obtenus dans les deux cas. Il apparaît que dans ces nouvelles conditions les concentrations respectives des trois acides aminés sont beaucoup plus élevées, bien que les concentrations initiales sous lesquelles ils sont ingérés soient identiques à celles se trouvant dans la lactalbumine. Il est évident que 1'absorption des acides aminés isolés est plus rapide (la concentration portale maximale est atteinte Io minutes après leur ingestion) et de plus grande amplitude que celle des protéines naturelles ; un phénomène d'interférence (PINSKY et GEIGER, I952; Hagihara et al., I960) ou de compétition pourrait être une explication hypothétique. A signaler également que la libération des acides aminés lors d'une digestion protéique étant relativement lente, cela pourrait fournir une réponse partielle aux différences existant entre les valeurs optimales obtenues avec des acides aminés isolés et avec des protéines.

Que ce soit par voie orale ou par voie parentérale, l'action de la déoxypyridoxine est plus nette sur les acides aminés isolés.

Il nous restait finalement à vérifier si des substances autres que les acides aminés peuvent avoir une influence sur la teneur portale de ces derniers. Pour cela, nous avons fourni aux rats simultanément du tryptophane et des concentrations croissantes d'amidon, et il a été possible de constater qu'à une concentration donnée, l'amidon trouble l'effet de la déoxypyridoxine.

Il faut remarquer qu'en commençant notre travail, nous avons admis implicitement que les fluctuations du niveau des acides aminés est en relation étroite avec le mécanisme de l'absorption; bien que logiquement cela paraisse exact, nous n'avons cependant pas assez de preuves pouvant confirmer cette supposition. Lorsque nous fournissons une protéine à un animal, nous admettons également qu'il existe un lien étroit entre les concentrations initiales des acides aminés de la protéine et la concentration de ces mêmes acides aminés dans le plasma portal. Bien que cela soit exact pour la plupart des acides aminés, il faut souligner certaines "anomalies" telles que celles mises en évidence par GoLDBERG et GuGGENHEIm (Ig62) pour la lysine et la méthionine.

Dans le cas particulier du tryptophane, la relation étroite existant entre l'action de la déoxypyridoxine et la concentration portale de cet acide aminé pourrait être en rapport avec le métabolisme du tryptophane, la pyridoxine intervenant directement dans son cycle métabolique ('THIERS, I956) et l'action antimétabolique de la déoxypyridoxine au niveau cellulaire se couplant avec son influence sur le transport de cet acide aminé.

Reçu pour publication en juin 1963.

\section{SUMMARY}

THE EFFECT OF DEOXYPYRIDOXINE ON PORTAL LEVELS OF AMINO ACIDS FOLLOWING A PROTEIN MEAL

The effect of deoxypyridoxine on the levels of lysine, methionine and tryptophane in the portal plasma has been studied in normal rats which had been given a test meal of either lactalbumine or of one of the above amino acids. It has been found that deoxypyridoxine affects the levels of lysine and tryptophane but has no effect on the methionine concentration. 
Pyridoxine deficiency depresses the levels of lysine and tryptophane in the portal blood; administration of pyridoxine does not abolish the effect of pyridoxine deficiency on tryptophane.

Starch added to tryptophane interfers with the effect of deoxypyridoxine on the tryptophane level of the portal plasma.

\section{RÉFÉRENCES BIBLIOGRAPHIQUES}

Agar W. T., Hird F. J. R., Sidhu G. S., 1953. The active absorption of amino acids by the intestine. J. Physiol., 121, $255^{-263}$.

Akedo H., Sugawa T., Yoshikawa S., I960. Intestinal absorption of amino acids. I. The effect of vitamin $\mathrm{B}_{6}$ on the absorption of $\mathrm{L}$-amino acids through the intestine. J. Biochem., Tokyo, $7,124^{-1} 3^{\circ}$.

Barton-Wright E. C., I952. The microbiological assay of the vitamin B Complex and amino acids, I2I-I 26. Isaac Pitman and Sons, London.

Cirristensen H. N., Riggs T. R., Coyne B. A., I954. Effects of pyridoxal and indoleacetate on cell uptake of amino acids and potassium. J. biol. Chem., 209, 4I 3-427.

Fridhandler L., Quastel J. H., 195.- Absorption of amino acids from isolated surviving intestine. Arch. Biochem. Biophys., 56, 424-440.

Goldberg A., Guggenheim K., I962. The digestive release of amino acids and their concentration in the portal plasma of rats after protein feeding. Biochem. J., 83, I 29-135.

Guggenheim K., Halevy S., Friedmann N., ig6o. Levels of lysine and methionine in portal blood of rats following protein feeding. Arch. Biochem. Biophys., 91, 6-ıo.

Hagihara H., Ogata M., Takedatsu M., Suda M., I960. Interference between amino acids during intestinal absorption. J. Biochem., Tokyo, 47, I39-143.

JACoBs F. A., rg62. Role of vitamin $B_{6}$ in intestinal absorption of amino acids in situ. J. Amer. med. Assoc., 179, $5^{2} 3^{-} 5^{2} 5$.

Jacobs F.A., Coen L. J., Hillman R. S. L., ig6o. Influence of pyridoxine, pyridoxal phosphate deoxypyridoxine and 2,4 dinitrophenol on methionine absorption. J. biol. Chem., 235, 1372-I375.

JaCobs F. A., FlaA R. C., BeLK W. F., I 960 . Pyridoxal phosphate requirement for intestinal absorption of L-tyrosine. J. biol. Chem., 235, 3224-3227.

JACoBs F. A., Luper M., 1957. Intestinal absorption by perfusion in situ. J. appl. Physiol., 11, 1 $3^{6-\mathrm{r}} 3^{8 .}$

Pinsky F. A., Geiger E., I952. Intestinal absorption of histidine as influenced by tryptophane in the rat. Proc. Soc. exp. Biol. Med., 81, 55-57.

Thiers H., 1956. In : Les Vitamines, 99-100. Masson et Cie. Librairie de l'Académie de Médecine, Paris. 